\title{
Anatomic relations of the arachnoidea around the pituitary stalk: relevance for surgical removal of craniopharyngiomas
}

\author{
Songtao Qi • Yuntao Lu • Jun Pan • Xi'an Zhang • \\ Hao Long • Jun Fan
}

Received: 26 June 2010 / Accepted: 31 December 2010 / Published online: 27 January 2011

(C) The Author(s) 2011. This article is published with open access at SpringerLink.com

\begin{abstract}
Purpose The growth pattern of craniopharyngiomas (CP) is yet to be understood due to challenges arising from the diversity of morphological features that exist. This in turn has had implications on the development of safe surgical strategies for management of these lesions. The aim of this study is to propose a morphological classification of $\mathrm{CP}$ based on their tumor-membrane relationship. It is hoped that this will contribute to better understanding of CP morphology and prediction of the intraoperative classification.

Methods Histological techniques were used to study eight fetuses. Following Masson staining, the membranes around the pituitary stalk were observed under microscope. Preoperative MRI and intraoperative images of 195 patients with $\mathrm{CP}$ were also analyzed.

Findings The arachnoidal sleeve around the pituitary stalk (ASPS) was noted to be comprised of a compact fibrous component and a related loose trabecular component. The pituitary stalk was divided into four segments in accordance with the folds of the ASPS. Correspondingly, the growth of CPs was divided into four basic patterns - infra-diaphragmatic (ID), extra-arachnoidal (EA), intra-arachnoidal (IA) and sub-arachnoidal (SA) growth. The IA growth pattern can be further subdivided into two subtypes - namely, IA1 (with tumor growing within the fibrous component of the ASPS) and IA2 (with tumor growing within the trabecular component). This method of topographical division can be
\end{abstract}

Yuntao Lu, M.D. contributes equally to this work. Yuntao Lu, M.D. is the co-first author of this article.

S. Qi $(\bowtie) \cdot$ Y. Lu $\cdot$ J. Pan $\cdot$ X. Zhang $\cdot$ H. Long $\cdot$ J. Fan

Department of Neurosurgery, Nanfang Hospital, Southern

Medical University,

Guangzhou 510515 Guangdong, China

e-mail: sjwk_songtao@hotmail.com used to understand the growth of $\mathrm{CP}$-infra-diaphragmatic CP show growth pattern ID or ID together with EA. Suprasellar CP can show an extra-ventricular growth pattern (EA or IA2), an extra- and intra-ventricular (IA2 +SA) growth pattern, a trans-infundibular growth pattern (ID+IA1+SA) and an infundibulo-tuberal growth pattern (SA or SA+IA1). There is a statistically significant difference between $\mathrm{CP}$ growth patterns in children and adults. A predominance of ID growth is noted in children while adults tend to show a pattern of predominantly Extra-ventricular (EV) growth.

Conclusion Our proposed classification details the relationship of the surrounding structures to CPs and purports to predict and identify the intraoperative anatomical stratification. It also attempts to help predict the growth patterns of these tumors. A knowledge of the intimate relations of the tumor and its key surrounding structures allows for safe surgical removal.

Keywords Arachnoid Classification .

Craniopharyngiomas $\cdot$ Membrane $\cdot$ Neurosurgery .

Pituitary stalk

\section{Introduction}

Craniopharyngiomas (CPs) arise from a remnant of the embryonic canal that stretches from the oropharynx to the suprasellar median eminence and infundibulum. In theory, any site along this vital axis might serve as the site of origin. The variable location and diversity of tumor extension contribute to innumerable growth patterns of CP. This, subsequently, further compounds the challenge of understanding the intraoperative tumor morphology. Partial removal is often undertaken to avoid the hypothalamic disturbance that usually accompanies total removal. 
However, the high recurrence rate of these lesions proves a challenge.

Various classification systems $[7,13,22,28,30]$ have been introduced to aid surgical planning. Within the literature on growth of $\mathrm{CPs}$, there is a variety of nomenclature used. Examples are: intra- or suprasellar tumor, extra- or intra-ventricular tumor (by Yasargil [31], Wang [29, 30] and Steno [28]), pre- or post-chiasmatic tumor (by Hoffman [7]), infra-chiasmatic tumor (Kitano [14]), or preinfundibular, trans-infundibular, or retroinfundibular tumor (Kassam [13]). These terms attempt to explain not only the tumor location, but also to aid in the choice of surgical approach. An issue which has been neglected within these existing classification systems is the tumor subtype. This can be highly challenging during tumor dissection and especially so, in the hands of an inexperienced neurosurgeon. The interface between tumor and surrounding vital structures is not always clear, even in expert hands.

In our preliminary work, the distribution of the membranous structures in the sellar region [26, 27] was depicted in detail, indicating that the pituitary stalk (PS) might have variant morphological features according to the envelope of the arachnoid sleeve [26]. Because of the close relation of $\mathrm{CP}$ to the PS, further analysis of the $\mathrm{CP}-$ membrane relationship was thought to be beneficial. Eight fetal specimens were subjected to histological analysis and 195 CPs presenting over the past 13 years were retrospectively analyzed. We proposed a morphological classification of CP based on the intraoperative tumor-membrane relationship observed. Moreover, by comparing the previous classification systems, the varying nomenclature of previous classifications were explained to better understand the tumor morphology and to predict the intraoperative stratification, and consequently to avoid injury of vital neural tissue during the tumor removal.

\section{Materials and methods}

Histological section

Heads from eight fetuses (age ranging from 6 to 8 months) were studied. Sellar region samples, including two parasellar CP and parts of the petrous apex were taken for serial histological sections coronally and sagittally, respectively (the slices being $8 \mu \mathrm{m}$ thick). Four out of every ten sections with important anatomical structures were selected for Masson's trichrome staining. An Olympus-DP70 microscope was used to observe the membranous structures. Histological analysis was carried out by Dr. Yuntao Lu (a dually qualified neurosurgeon and neuropathologist).
Clinical analysis

In order to find a correlation between the membranous structures of this region and the growth pattern of CP, 195 cases of CPs were selected for clinical analysis (from January 1996 until the present). The inclusion criteria for this cohort were:

1. Primary surgery. Patients with primary surgery have intact sellar anatomy construction, which will be destroyed after operation;

2. Adequate pre- and post-operative radiology and intraoperative imaging. MRIs and CTs (axial, coronal, and saggital; FLAIR and contrast-enhanced) were analyzed by Dr. Jun Pan. All operations (by Professor Songtao Qi) were recorded in video format, and some intraoperative images with textbook features were used to demonstrate the tumor-membrane relationship.

\section{Statistically comparison}

The growth patterns of CPs in adults and children were statistically analyzed and described, respectively. In order to investigate the variant distribution of the growth pattern, two-sample independent test of statistical significance (Mann-Whitney test) was used to compare the adult and children cases (using SPSS 15.0 software). Statistical significance was set at a $p$ value $<0.05$.

\section{Results}

ASPS and four segments of pituitary stalk

On the sagittal sections through the PS, it was noted that the suprasellar arachnoid arising from the basement membrane of the arachnoid (BAM) enveloped the pituitary stalk to form an arachnoidal sleeve (ASPS). The ASPS was observed to comprise two parts-a fibrous component and a trabecular component (Fig. 1). From the coronal sections, the ASPS was noted to be tightly adherent to the pia mater covering the external surface of optic chiasm. This rendered histological differentiation of these structures to be a challenge. The distal part of the PS was found located beneath the ASPS without envelopment of the arachnoidal sleeve. The magnified coronal section depicted in Fig. 1d demonstrates that the whole PS can be divided into four segments - the infra-diaphragmatic, extra-arachnoidal, intra-arachnoidal, and sub-arachnoidal segments.

\section{Classification of the CP's growth pattern}

The growth pattern of $\mathrm{CP}$ was divided into four subsets based on tumor location, relationship with arachnoidal 


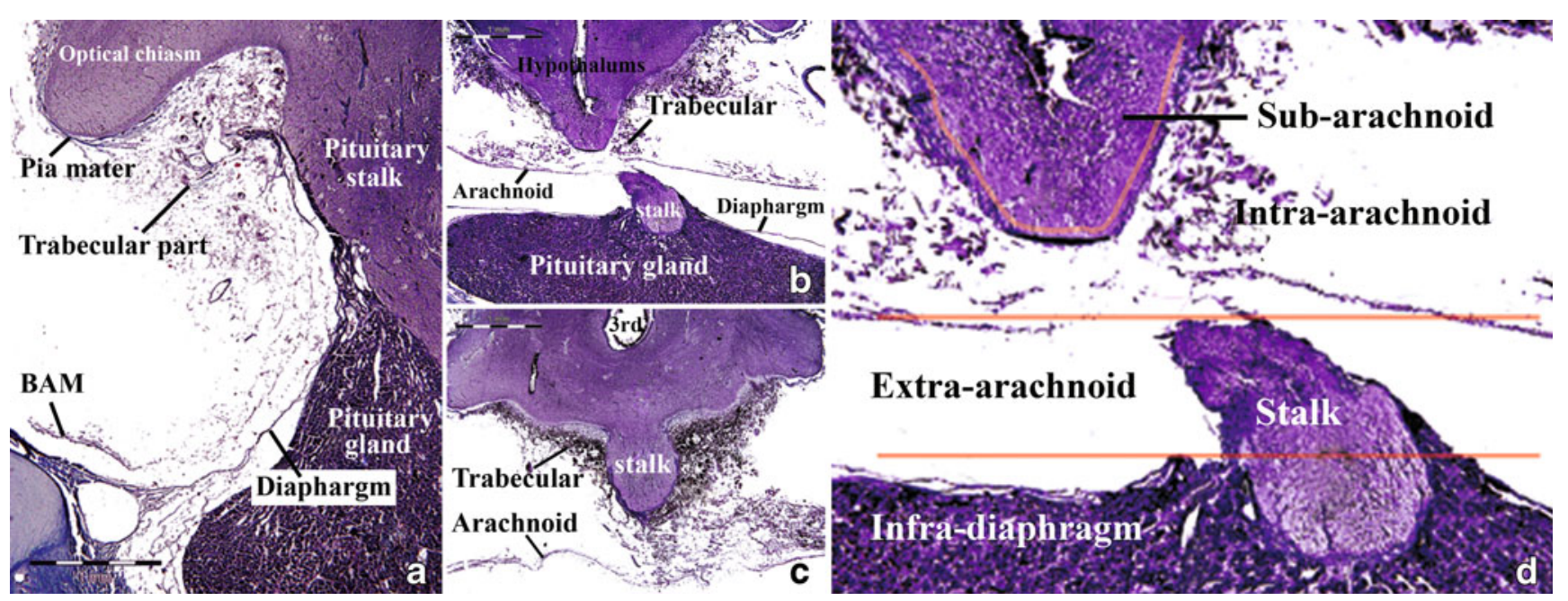

Fig. 1 Sagittal and coronal section through PS showing the ASPS and the segmentation of the PS (Masson trichrome stain). a, b, $\mathbf{c}$ Being continuous with BAM, the suprasellar arachnoid wrapped the PS and formed the fibrous part of ASPS. With reinforcement by the trabecular

structures, thorough analysis of pre-operative MRI, as well as intraoperative findings of the $195 \mathrm{CPs}$. The four basic types were: the infra-diaphragmatic (ID), extra-arachnoidal (EA), intra-arachnoidal (IA), and sub-arachnoidal growth (SA; as depicted in Fig. 2). The possible site of origin of CP with variant growth patterns is also depicted in this figure.

Pattern ID With this subtype, the entire tumor is encased by the dura mater and the site of origin is most likely to be beneath the diaphragm. The suprasellar arachnoid courses beyond the upper surface of diaphragm. The optic chiasm and the ASPS are pushed posteriorly and superiorly by tumor, thereby creating a big prechiasmatic space (first space). part, ASPS tends to thicken at the ventral aspect of optic chiasm; and ASPS also adhere tightly to the pia mater. $\mathbf{d}$ The amplification view showing the whole PS can be divided into: infra-diaphragmatic, extraarachnoidal, intra-arachnoidal, and sub-arachnoidal segment

Pattern EA The tumoral site of origin was probably located at the extra-arachnoidal segment of the PS. With external growth, tumor inevitably encroached on the extremely enlarged first space. Lacking a diaphragmatic covering, the tumor is in direct contact with the suprasellar arachnoid. This provides an interface to separate tumor from optic chiasm and the tubero-infundibulum.

Pattern IA Tumor originates from the intra-arachnoidal segment of the PS, with expansion within the ASPS. This can be further subdivided into two subtypes: IA1 and IA2. With IA1, tumor grows within the fibrous part of the ASPS, causing enlargement of the PS. The diaphragm is pushed downwards, while the optic chiasm

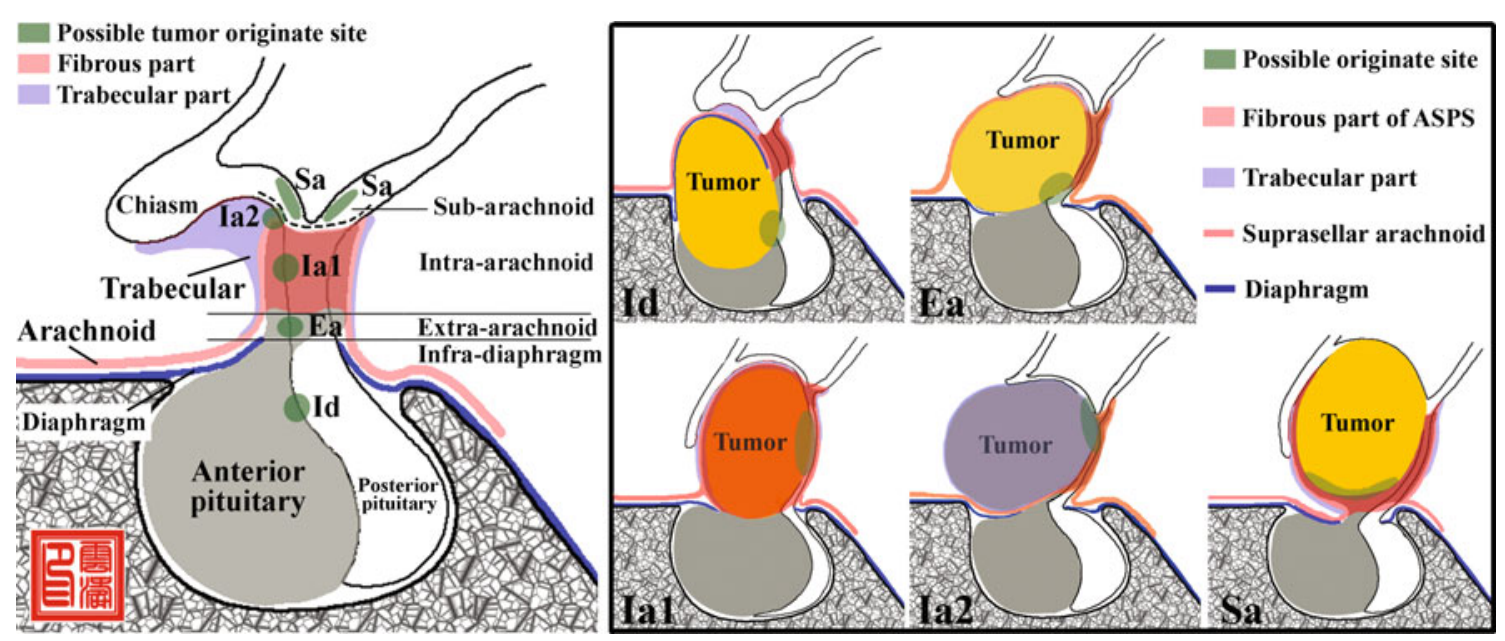

Fig. 2 The schematic diagram shows the four basic growth patterns of CP. The left figure shows ASPS and the four segments of the stalk. Correspondingly, several different possible tumor originating points were indicated as "Id, Ea, Ia1, Ia2, and Sa", which were in correspondence to the basic tumor growth patterns shown in the right figure 
is displaced anteriorly, resulting in a comparatively small first space. Tumor showing IA2 growth pattern, grow mainly within the trabecular part of ASPS, displacing the PS posteriorly and the suprasellar arachnoid inferiorly. Some tumors will also expand prechiasmatically resulting in an enlarged first space.

Pattern SA With the probable site of origin being the tubero-infundibulum, these tumors growth within the third ventricular floor (third VF) and are entirely located within the sub-arachnoidal space, displacing the ASPS inferiorly. These tumors tend to involve the hypothalamus and there is often a lack of any membranous plane for separation.

\section{Clinical analysis}

The pre-operative imaging and intraoperative videos of 195 cases of CPs in this cohort were used to depict the tumor growth as illustrated in Figs. 3, 4, 5, 6, 7, 8 and 9. For purposes of clarity, the various structures are delineated in pre-operative imaging using the respective color codes - dura mater (blue), diaphragm (yellow), suprasellar arachnoid (pink), and ASPS (fibrous part—pink area; trabecular part—blue area).

(A). Infra-diaphragmatic tumor. Pattern "ID" is the basic growth pattern observed with CP (Fig. 3). In some cases however, $\mathrm{CP}$ can protrude through the enlarged diaphragmatic opening, and grow extra-arachnoidally, thus presenting with the pattern, "ID+EA" (Fig. 4).

(B). Suprasellar extra-ventricular tumor. Corresponding to either a lower or upper origin from the PS, this tumor type can have two different basic growth patterns. With a lower site of origin (the extra-arachnoidal segment of PS), tumor grew beneath the fibrous arachnoid and the ASPS, hence presenting as pattern "EA" (Fig. 5). On the other hand, with an upper site of origin (the proximal part of the intra-arachnoidal segment), tumor grew within the trabecular component of the ASPS displacing the fibrous arachnoid downwards, hence presenting as growth pattern "IA2" (Fig. 6, see the description of the extraventricular part of tumor).

(C). Extra- and intra-ventricular tumor. In some cases, extra-ventricular tumors with pattern "IA2" can also demonstrate a sub-arachnoidal growth (pattern "SA"), thereby presenting a combined pattern of extra- and intra-ventricular growth (pattern "IA2+ SA"; Fig. 6).

(D). Trans-infundibular tumor. When the site of origin is from the intra-arachnoidal segment of the PS, CP tends to expand within the stalk covered by the fibrous part of ASPS (pattern "IA1") superiorly and
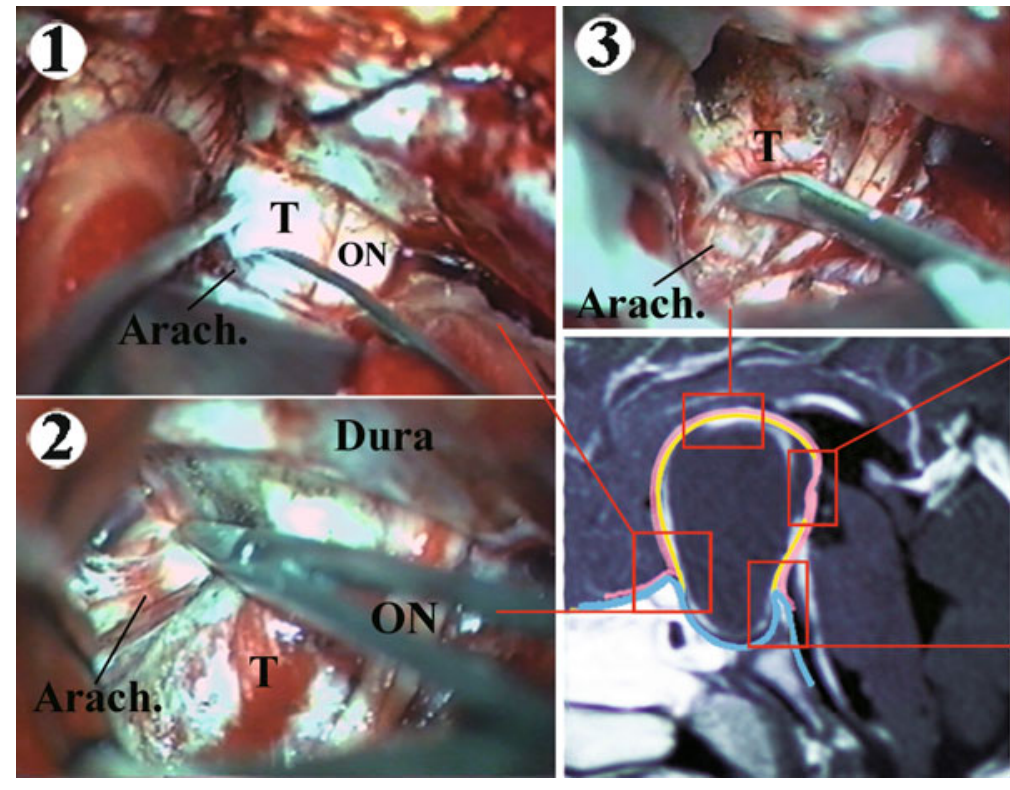

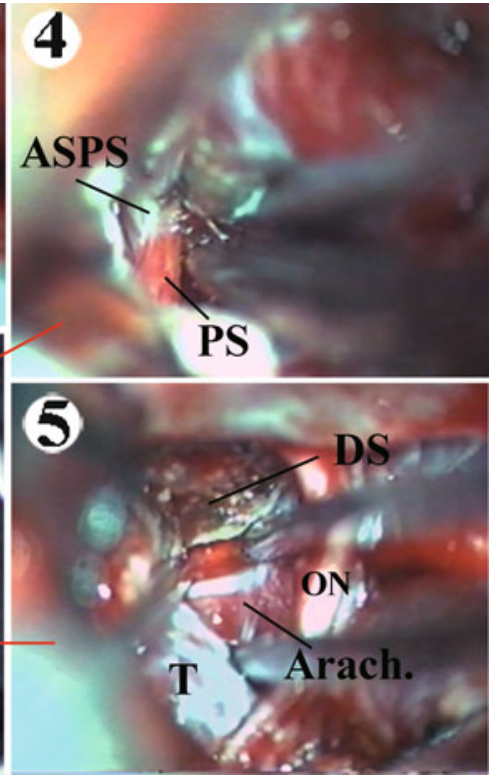

Fig. 3 Infra-diaphragmatic CP with single growth pattern "Id". In the presurgical MRI (sagittal), a cystic CP was found being located intra and suprasellar region, with enhanced signal of the tumor wall. By constant pushing of the tumor, the diaphragm was presenting as an egg wall shape and covering the whole tumor. With severe involvement of sellae, the pituitary gland and stalk could not be clearly identified. Intra-operative image: as the view of the frontointerhemispheric approach, a quite big prechiasmatic space was found in most of this type of tumors (1). Through this space, a smooth tumor's wall $(T)$ seemed to continue with the dura mater covering the planum sphenoidale (2). And a thin layer of arachnoid (Arach.) was found covering the surface of tumor $(2,3)$. Behind the tumor, the upper one-third of PS can be found wrapping by the ASPS $(4,5)$, while because of extremely expanding of tumor, the lower two-thirds was seldom clearly identified. $O N$ optic nerve, $D S$ dorsum sellae 

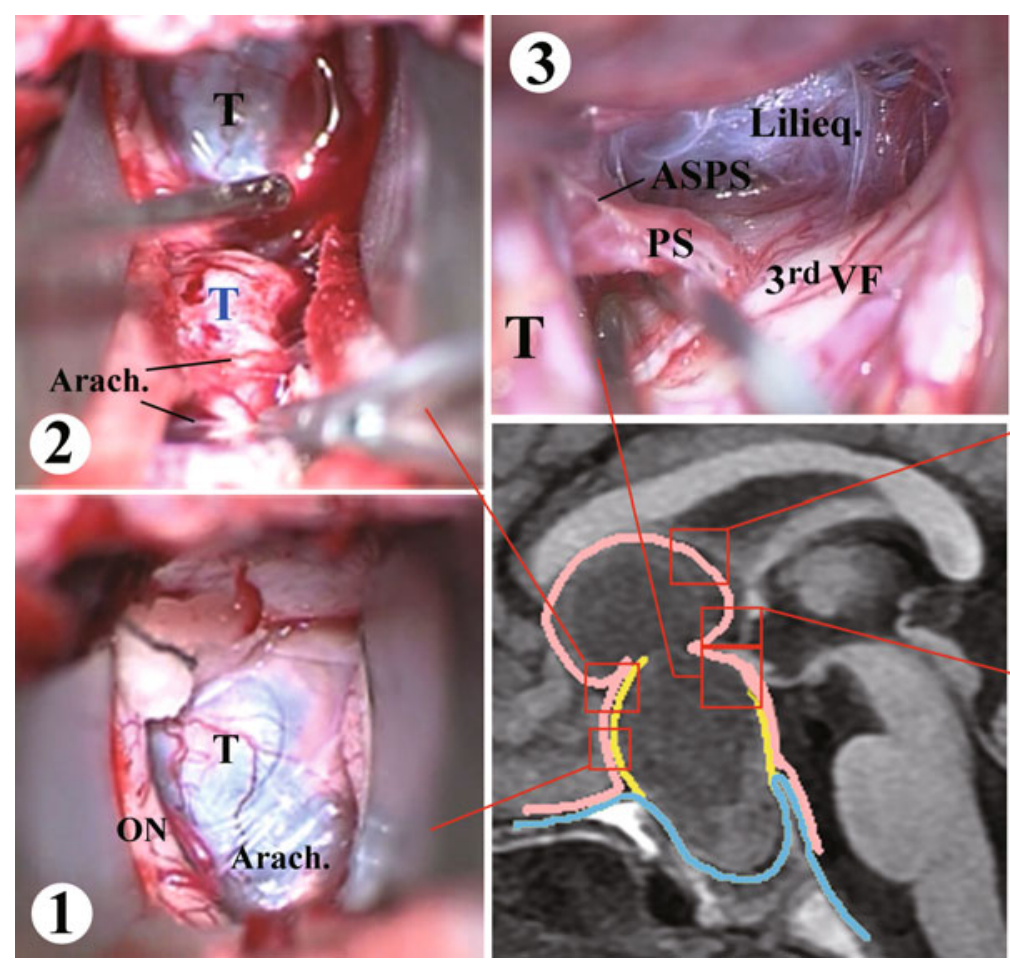
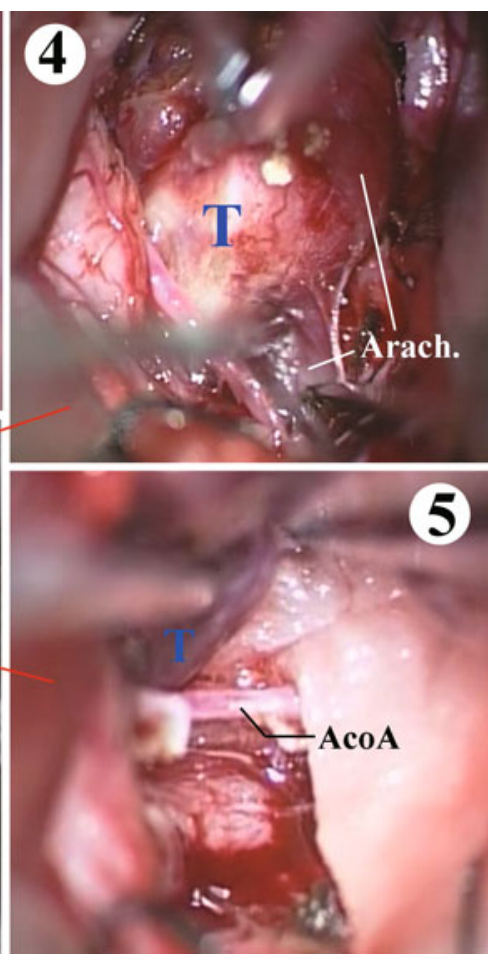

Fig. 4 Infra-diaphragmatic CP with pattern "Id+Ea". Same as the tumor with single pattern "Id" (Fig. 3), through the enlarged prechiasmatic space, a smooth tumor (black T) wall seemed to continue with the dura mater covering the planum sphenoidale (1). A layer of arachnoid (Arach.) was found covering the surface of it (pattern "Id"); while part of tumor protrudes through the diaphragmatic opening (blue T), and with the restriction of which, formed a snowman sign (2) in the presurgical MRI. In these cases, the tumor part beyond the diaphragm was also covered by the arachnoid (pattern "Ea") (4), which also separates it from the optic chiasm and the AcoA as indicated by the (5). The ASPS was identified wrapping the PS as well. Meanwhile, the proximal part of PS and the third VF was always intact and can be protected well (3). Commonly, the pituitary gland and the distal part of stalk can hardly be identified. $O N$ optic nerve, Lilieq. Liliequist membrane inferiorly. This in turn causes the stalk to bulge and expand (Fig. 7). CP can expand not only to the subarachnoidal area (pattern "SA"), but also within the pituitary fossa by penetrating through the diaphragm. The intrasellar part of tumor is then covered by the diaphragm (pattern "ID"). The resulting growth pattern is, "ID+IA1+SA".

(E). Infundibulo-tuberal tumor. This tumor appears to originate from the sub-arachnoidal segment of the PS and always presents growth pattern "SA" (Fig. 8). There is significant deformation of the third VF with subsequent growth of the CP. There is also the possibility of hydrocephalus, secondary to obstruction of the foramen of Monroe. Aside from the straightforward growth pattern depicted in "SA", infundibulo-tuberal $\mathrm{CP}$ can also expand within the fibrous part of the ASPS leading to growth pattern, "SA+IA1" (Fig. 9). As tumors with these growth patterns are different to trans-fundibular tumors, the infra-diaphragmatic growth (pattern "ID") was not noted in this subgroup.

\section{Comparison of cases between adults and children}

One hundred and ninety-five cases of CPs were statistically analyzed (as shown in Table 1, demonstrating the distribution of tumors with different growth patterns in adults and children). Children showed a significantly different growth pattern compared to adults with CP ( $p=$ 0.023). Infra-diaphragmatic CPs were predominantly observed in children rather than adults $(42 \%$ vs. $7.0 \%, p<$ $0.0001)$. In contrast, almost all extra-ventricular tumors were observed in adult patients $(28.1 \%$ vs. $3.7 \%, p<0.0001)$. However, there was no significant difference in the distribution of extra- and intra-ventricular tumors $(4.4 \%$ vs. $6.2 \%, p=0.578)$ or trans-infundibular tumors $(2.6 \%$ vs. $1.3 \%, p=0.499$ ).

Interestingly, with regards to infundibulo-tuberal CPs, although no significant difference was found between adults and children $(57.9 \%$ vs. $46.8 \%, p=0.131)$, the tumors with pure pattern "SA" showed predominance in adults $(47.4 \%$ vs. $30.8 \%, p=0.021)$. A $p$ value of 0.257 however indicated no statistical significance in the difference 

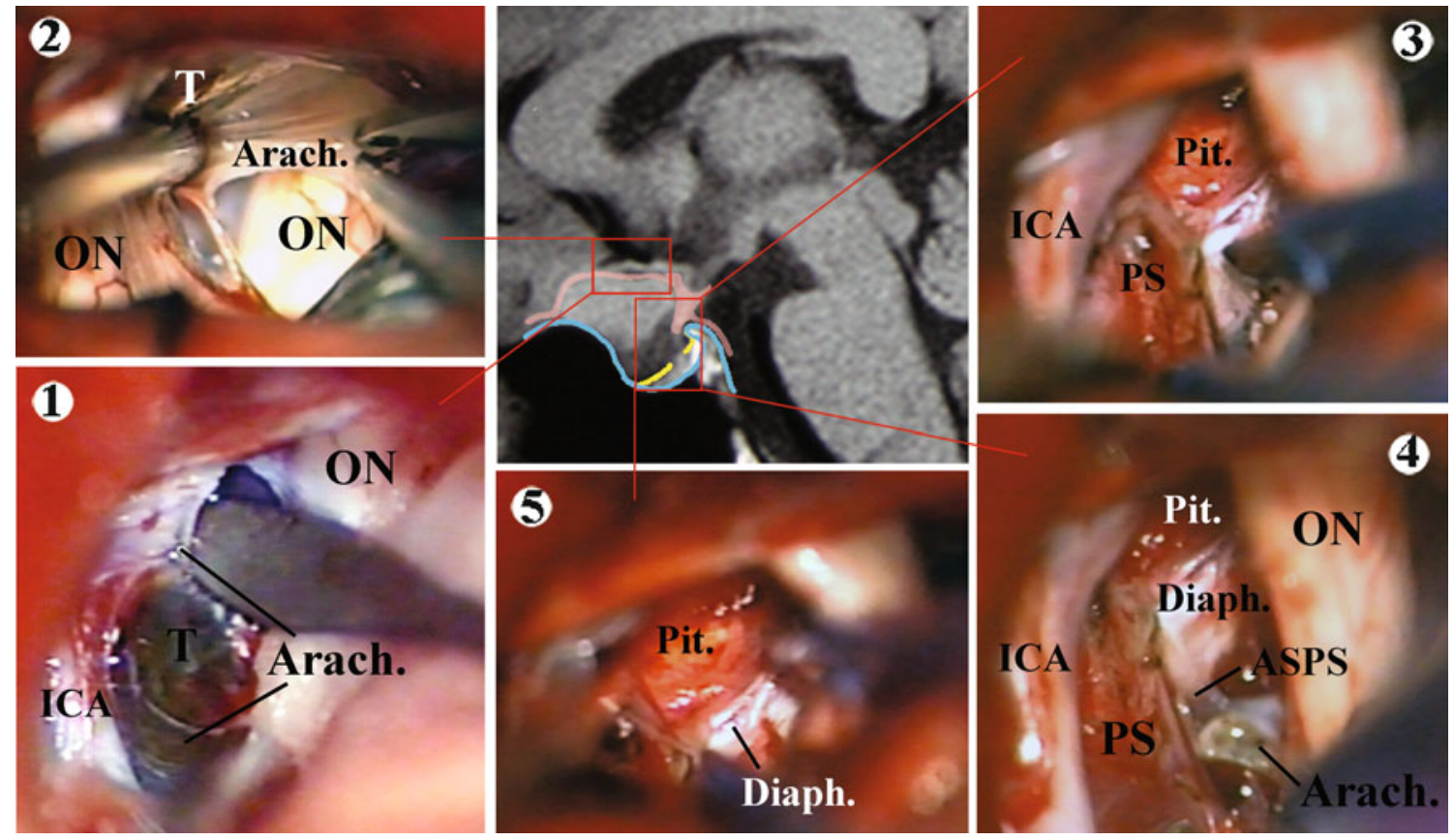

Fig. 5 The extra-ventricular CP with growth "Ea". In the presurgical MRI T1, an intra- and suprasellar tumor was found. Prechiasmatic growth and the intact third VF indicated the extra-ventricular tumor type. The tumor grows with protruding outside the PS and totally extra the arachnoidal cistern. The stalk was pushed backwards, and the normal gland can be identified at the bottom of the hypophyseal fossa with covering by the diaphragm. Intraoperative images: with a left pterional approach, through the enlarged first space, a fibrous

in distribution of the infundibulo-tuberal tumors with pattern "IA+SA" in adults and children.

\section{Discussion}

Arachnoidal sleeve of the pituitary stalk

Since their initial discovery by Gerardus in 1666 and subsequent naming by Frederick in 1692, arachnoidal sleeves have been studied for more than 300 years. Yasargil described the detailed anatomy of the sub-arachnoidal cisterns in his landmark studies published in 1976 and 1984 [21, 32]. The arachnoid of the sellar region has been of especial interest in recent years due to complexities and vital structures involved. Correspondingly, the cisterns composed by these membranes, such as the chiasmatic, carotid, and prepontine cisterns have been welldocumented. Located close to the PS, the chiasmatic cistern has traditionally been a popular corridor to be utilized during surgery. As recently described by Jian L [20], the chiasmatic membrane is composed of two leaves - the suprachiasmatic leaf and basal leaf. The former extends posterosuperiorly along the superior surface of the optic chiasm. The latter is located below the optic chiasm and arachnoid (Arachnoid.) was found covering the surface of the tumor (T) (2), which also separate the tumor from optic nerves and ICA (1). The blunt dissection can be used to free it. At the bottom of the tumor, the downwards displaced diaphragm (Diaph.) and enlarged diaphragmatic opening were found $(3,5)$. A relative intact PS was found wrapped by ASPS, which being continuous with the arachnoidal membrane (4). Generally, the continuity of the third VF-stalkpituitary gland (Pit.) can be protected totally. $O N$ optic nerve

spreads posteriorly against the surface of the diaphragm. The basal leaf extends from the ventral aspect of the chiasm to the PS and then onto the surface of the diaphragm. The concept of an outer and inner component to the arachnoid membrane was proposed by Inoue et al. [10] in their further studies of the chiasmatic cistern. According to this, the outer component was described as surrounding the entire brain and the inner component divided the subarachnoid space into cisterns. Two medial carotid membranes, one diencephalic (inner arachnoid) and the outer arachnoid resting on the diaphragm, are thought to comprise the walls of the chiasmatic cistern. Although the PS has been described as being located within this cistern in all previously published studies, the arachnoid membrane surrounding it has been relatively neglected.

In our last paper [26], we studied 21 adult cadavers anatomically and histologically. We found an arachnoidal sleeve wrapping the PS (ASPS), arising from the BAM (outer arachnoid) and ending at the pars tuberalis. The fetal specimens described in this study were noted to have an ASPS that was not only composed of the outer arachnoid (fibrous part), but also an inner arachnoid (trabecular part). The outer layer covers the tuberculum and diaphragm, coursing towards PS and wrapping the stalk superiorly. The trabecular arachnoid lies at the ventral aspect of the optic 

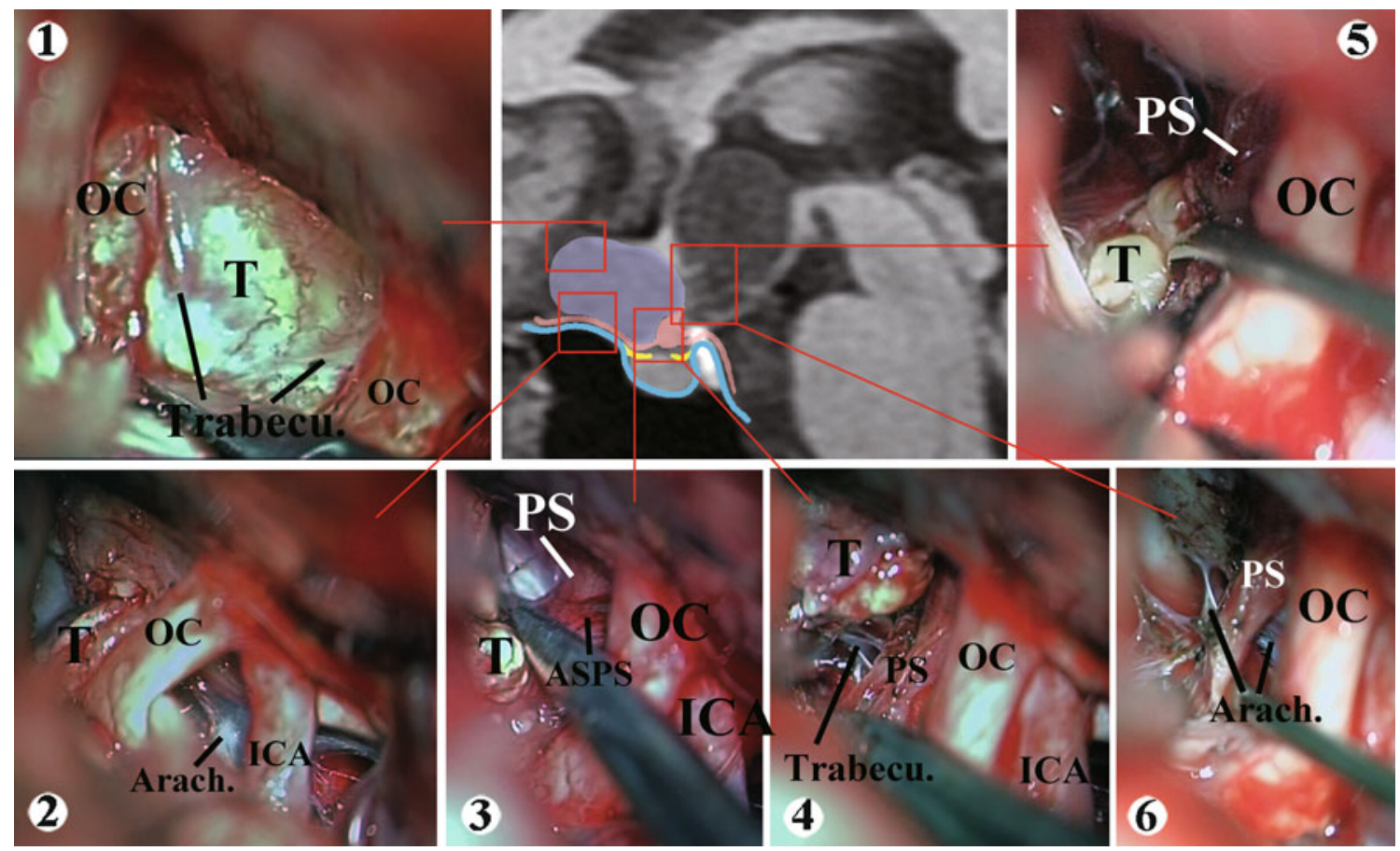

Fig. $6 \mathrm{CP}$ with extra- and intra-ventricular tumor $(\mathrm{Ia} 2+\mathrm{Sa})$. In the presurgical MRI-T1, prechiasmatic growth and the identifiable PS indicated the extra-ventricular growth of the front tumor part (pattern "Ia2"). The inflated infundibulum and third VF also shows the intraventricular part tumor (pattern "Sa"). The blue area indicated the trabecular part of ASPS, which covers the extra-ventricular tumor's surface. Intraoperative images: through the enlarged first space, tumor was found being wrapped by a layer of relative loose membranous trabeculum (Trabecu.). Even with the separation of these trabeculae, the tumor always adhered to the neurovascular structure tightly, which

chiasm and is thought to be connected with the lamina terminalis and medial carotid membrane. This inner arachnoid wraps the pituitary stalk inferiorly. The PS is thus well protected by both these membranes.

The four basic growth patterns of $\mathrm{CP}$

In reviewing the existing literature, the classification systems of CP can be generally divided into two categories: (1) those based on horizontal extension (such as Hoffman et al. $[7,8]$ ) which classify $\mathrm{CP}$ with respect to the sella turcica, optic chiasm, and the floor of the third ventricle, into prechiasmatic, retrochiasmatic, subchiasmatic, and intraventricular CPs, and (2) those based on vertical extension (such as Sammi et al.) which classify CPs into Grade I (intrasellar or infra-diaphragmatic), II (occupying the cistern with or without an intrasellar component), III (lower half of the third ventricle), IV (upper half of the third ventricle), and $\mathrm{V}$ (reaching the septum pellucidum or lateral ventricles) [24, 25]. However, in practice, CPs with a diverse range of extension patterns may give rise to similar MRI appearances. Examples include tubero-infundibular must be freed by the sharp dissection (1). Beneath of the tumor, a layer of intact fibrous arachnoid was found, which being continuous with the ASPS wrapping the PS $(2,3)$. Near the third VF and at the top of the extra-ventricular tumor part, some trabeculae still separate tumor and nerves tissue (4). After removing the tumor inside the trabecular part of ASPS, an inflated third VF can be found. After incising it (5), the intra-ventricular tumor was exposed and found being with no membranous separation between tumor and the nervous structure. With total tumor removal, the continuity of the third VFstalk- pituitary gland was partly protected (6). $O N$ optic nerve

tumors with inferior extension which can involve the intrasellar region, and in comparison, infra-diaphragmatic tumors with superior extension that can also displace the third VF. Additionally, some large tumors with extension to the anterior skull base or the interpeduncular cistern, actually have a relatively limited base at the PS, allowing adequate preservation following total tumor resection. Normal-sized tumors located totally within the pituitary stalk or third VF will pose much more of a challenge when trying to preserve the integrity of these structures. All these problems and confusion about the true growth model of CPs has led us to devise a supplement classification of the previous systems [7, 13, 22, 28-31].

Based on our preliminary work on the distribution of membranous structures within the sellar region, we propose a topographic classification based on the unique tumorarachnoid relationship, which was directly correlated with the surgical stratification. It can also clearly delineate for neurosurgeons, the interaction of tumor and surrounding structures. Correspondingly, the membranes also provide a different surgical interface for each kind of tumor growth pattern. This in turn directly determines the surgical 

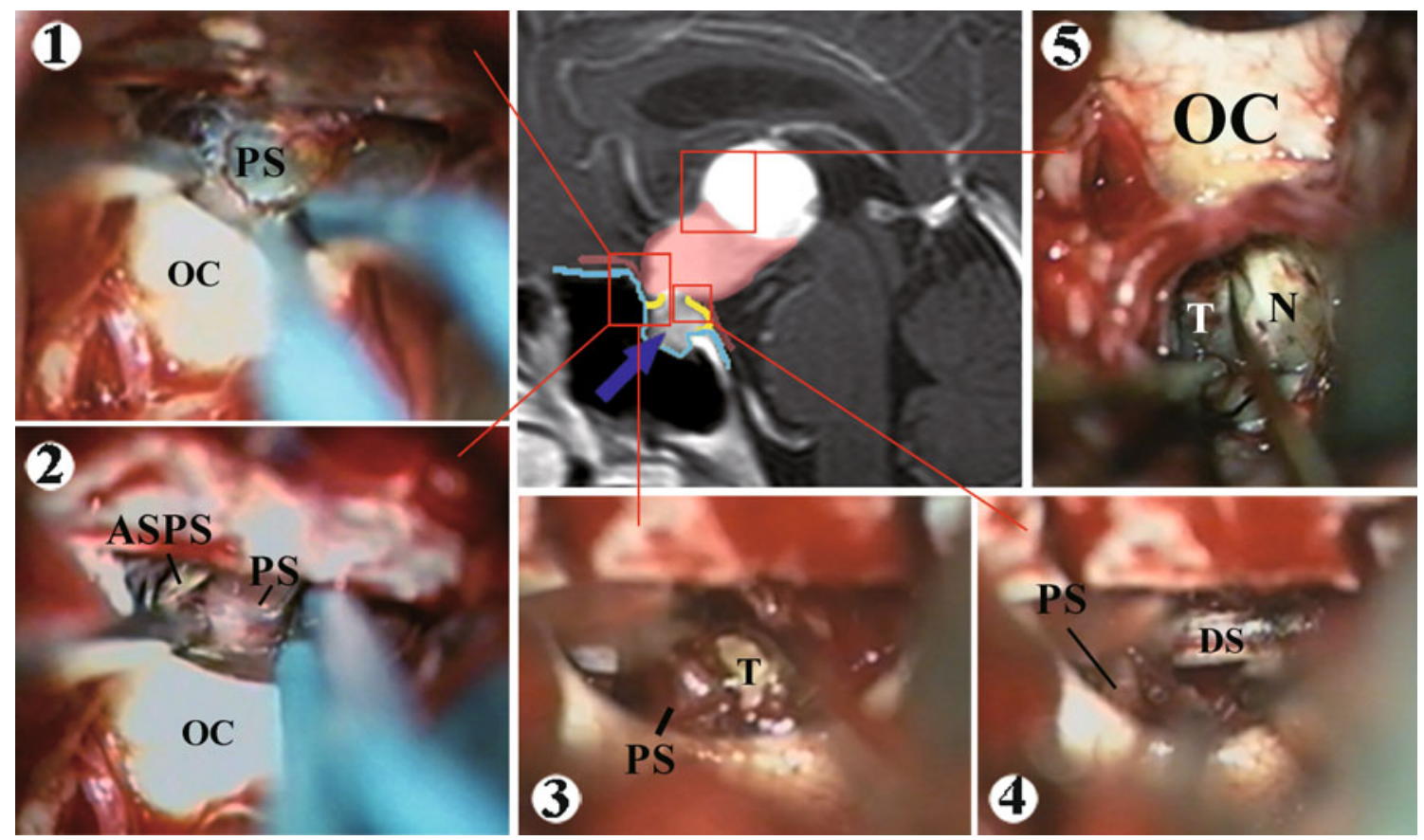

Fig. 7 Trans-infundibular $\mathrm{CP}$ with growth pattern "Id+Ia1+Sa". Presurgical enhanced MRI shows an intra-suprasellar tumor with Mickey Mouse shape. With the expanding growth inside the PS, tumor formed in the intrasellar part (blue arrow) and suprasellar part. The diaphragm covers the intrasellar part (pattern "Id"), but not the suprasellar tumor. The normal pituitary cannot be identified. The fibrous ASPS enveloped the lower part of suprasellar tumor ("Ia1"), which separate tumor with the surrounding structures. While at the upper pole, the intra-ventricular par of tumor located inside the third VF directly without separate by arachnoid ("Sa"); Intraoperative

technique and the feasibility of preservation of the PS and third VF.

With ID growth, tumor tends to be covered by dura mater and also by arachnoid outside the diaphragm, which thus separates tumor distinctly from all the suprasellar structures. Hence, blunt dissection can be used to free the tumor from the suprasellar neurovascular structures. However, due to the tumor's intrasellar location, the diaphragm needs to be incised to expose the tumor. In this type of tumor, the proximal one-third of the stalk was always noted to be intact. In some cases, the fibrous stalk could be identified posterior to the tumor, and attempts were made to preserve this. According to tumor morphology and location, the transsphenoidal approach is the most appropriate corridor to treat the tumor. This is due to the protection afforded by the diaphragm and the suprasellar arachnoid in avoiding injury to vital structures (such as the optic chiasm and third VF).

Tumor demonstrating EA growth was noted to be covered by relatively compact fibrous arachnoid. This provided a surgical interface between tumor and surrounding structures. In general, a relatively intact PS could be identified posterior to the tumor. The continuity of the third images: through an almost normal size of first space, an expanded PS was observed being wrapped by the ASPS $(1,2)$. The proximal part of stalk bulged with a reversed umbrella-shaped. After incising the inflated PS, tumor can be exposed being insides the stalk (3). The border of diaphragm can also be identified (4). For removing the intraventricular tumor, the lamina terminalis space always should be used. This part of tumor was found growing inside the third VF without any arachnoidal separation. A thin nervous tissue layer $(N)$ still covered the surface of the tumor $(T)(5)$. $O C$ optic chiasm; $D S$ dorsum sellae

VF-stalk-pituitary gland can be well protected in this type of tumor.

The above two types of tumors were thought to originate from the lower part of the PS and can be identified as directly connecting with the stalk. Tumors showing IA growth comprised two different scenarios. Pattern IA1 consisted of tumor growing within the fibrous part of the ASPS with a median point of origin. Subsequent growth resulted in expansion of the PS. In this scenario, although the ASPS can separate tumor from surrounding structures, the stalk had to be vertically dissected to remove the tumor. The other scenario is that of tumor originating in the upper portion. The relatively loose trabecular part of ASPS results in tumor presenting as a protruding growth outside the stalk and within the trabeculae (pattern IA2). The tumor can adhere to different extents to the surrounding neurovascular structures necessitating sharp dissection to free up structures.

Of the three examples described above, the extra-axial approach is the optimal surgical option to avoid injury of the third VF. This is because the tumor is still separated from the sub-arachnoidal area by membranous structures. On the contrary, for tumor showing SA growth, the intra- 

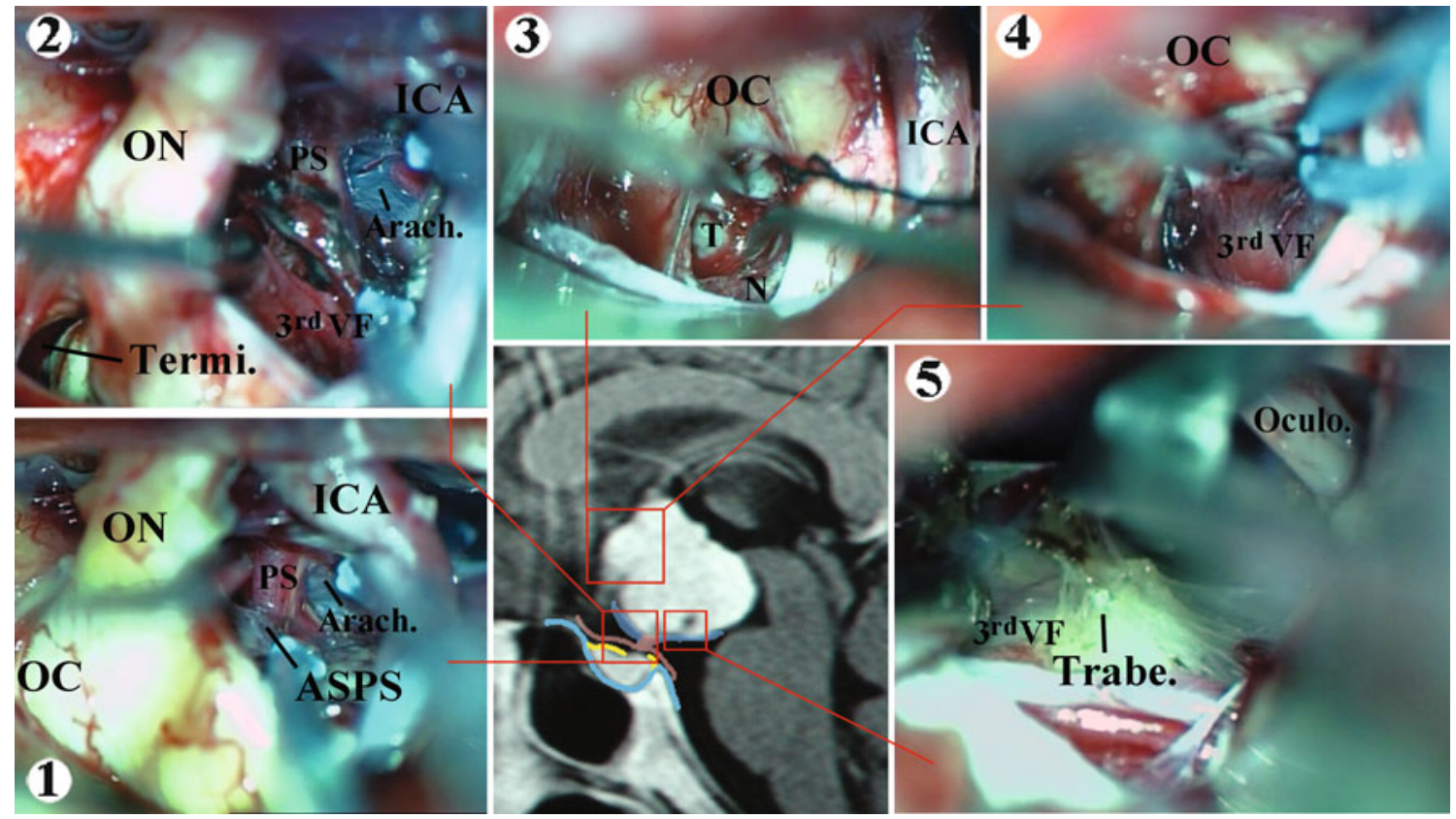

Fig. 8 The infundibulo-tuberal CP with single growth pattern "Sa". In presurgical T1-W, a suprasellar tumor was found causing the replacement of the third VF. The distal part of the stalk can be identified to connect with the normal gland, which was enveloped by the ASPS. The diaphragm was marked, which still sealed the pituitary fossa. The blue area shows the trabecular part of ASPS. In operation, a small first space was found in most of this type of tumors (1). Through the optic-ICA space (second space), an intact distal part of stalk was always found in this type of tumors, which was wrapping by a thicken ASPS (1). The

inflated and bulged third VF with a frog-belly shape was observed being covered by some trabeculae (Trabe,; 5). By lamina terminalis opening (Termi.), the tumor $(T)$ contacts with the nervous tissue layer $(N)$ directly without membranous separation (3). After tumor removal, the residual third VF can be identified at one lateral side (4); the incision of the third VF was also shown. The continuity of third VF-stalk-pituitary gland was protected partly (2). While in some cases with huge calcification, the continuity has to be amputated because of extremely thin third VF. $O N$ optic nerve; $O C$ optic chiasm
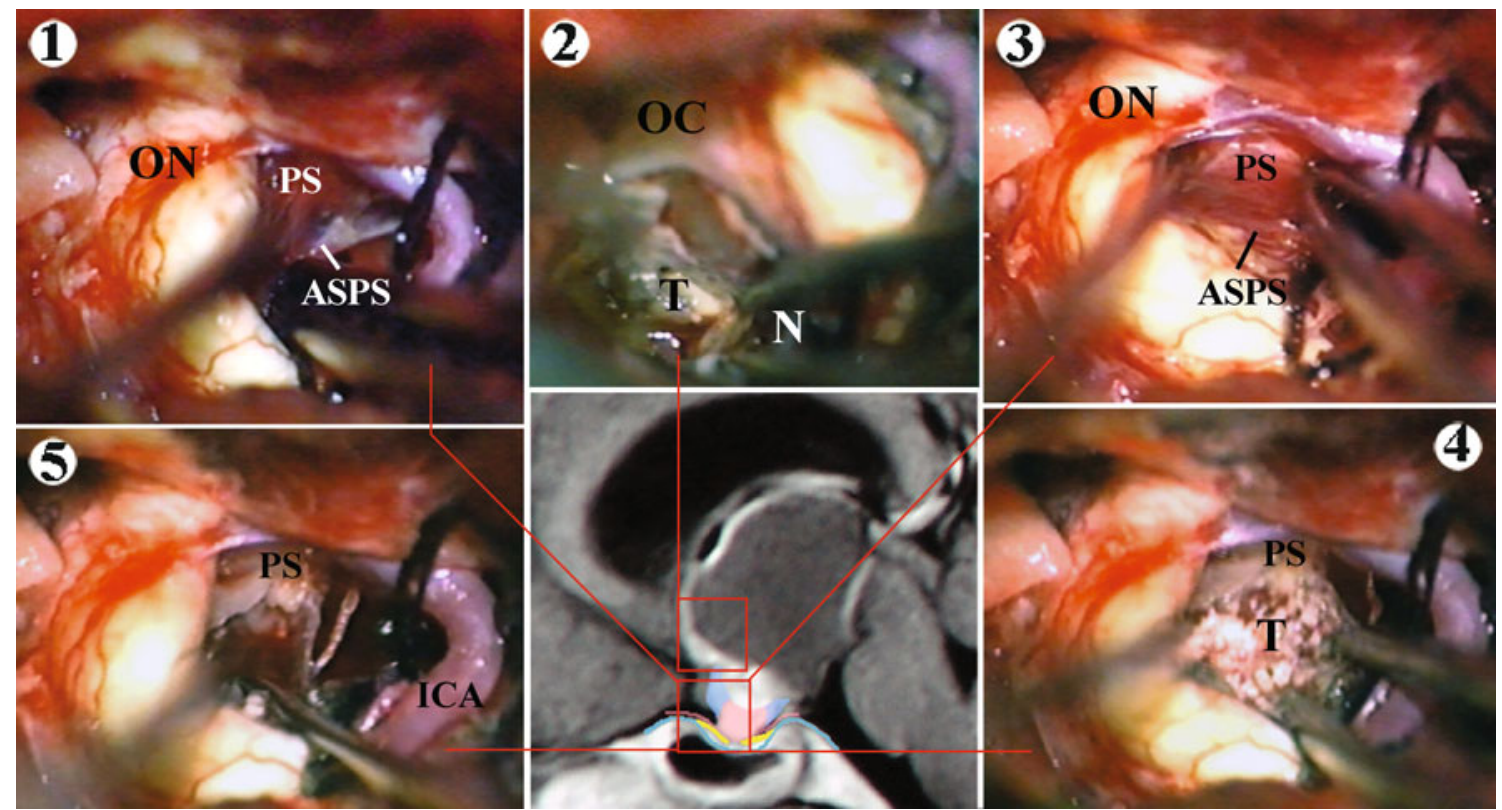

Fig. 9 The infundibulo-tuberal $\mathrm{CP}$ with growth pattern "Ia1+Sa". In presurgical MRI T1, an intra-ventricular CP was found causing severe hydrocephalus. The diaphragm and normal pituitary gland were pushed downwards and located in the bottom of hypophyseal fossa, with result in the sellar involvement in MRI. Tumor grew towards the third VF behind the optic chiasm, which cause a quite small first space. Intraoperative images: through the second space, an inflated PS wrapped by ASPS can be identified $(1,3)$. The lamina terminalis space had to be opened to remove the intra-ventricular part of tumor, the tumor $(T)$ contact with nervous tissue layer $(N)$ without membranous separation (2). Then, a vertical incision of PS had to be done to expose the tumor inside the stalk (4). After tumor's removal, the distal pole of stalk was found being intact (5). $O N$ Optical nerve 
Table 1 The clinical cases analysis of the 195 craniopharyngiomas

\begin{tabular}{|c|c|c|c|c|c|c|}
\hline \multicolumn{2}{|c|}{ No. of $\mathrm{CP}$ with the growth pattern being present } & \multicolumn{2}{|c|}{ Adult $^{\mathrm{a}}$} & \multicolumn{2}{|c|}{ Child } & \multirow[t]{2}{*}{ Total } \\
\hline & & $n$ & $\%$ & $n$ & $\%$ & \\
\hline Infra-diaphragm $^{\mathrm{b}}$ & $\begin{array}{l}\text { Pattern "Id" } \\
\text { Pattern "Id+Ea" }\end{array}$ & $\begin{array}{l}8 \\
0\end{array}$ & $\begin{array}{l}7.0 \% \\
0 \%\end{array}$ & $\begin{array}{l}25 \\
9\end{array}$ & $\begin{array}{l}30.8 \% \\
11.2 \%\end{array}$ & 42 \\
\hline Extra-ventricular ${ }^{\mathrm{c}}$ & $\begin{array}{l}\text { Pattern "Ea" } \\
\text { Pattern "Ia2" }\end{array}$ & $\begin{array}{l}24 \\
8\end{array}$ & $\begin{array}{r}21.1 \% \\
7.0 \%\end{array}$ & $\begin{array}{l}3 \\
0\end{array}$ & $\begin{array}{l}3.7 \% \\
0 \%\end{array}$ & 35 \\
\hline Extra- and intra-ventricular & Pattern "Ia2+Sa" & 5 & $4.4 \%$ & 5 & $6.2 \%$ & 10 \\
\hline Trans-infundibular & Pattern "Id+Ia1+Sa" & 3 & $2.6 \%$ & 1 & $1.3 \%$ & 4 \\
\hline Infundibulo-tuberal & $\begin{array}{l}\text { Pattern "Sa" } \\
\text { Pattern "Ia1+Sa" }\end{array}$ & $\begin{array}{l}54^{\mathrm{d}} \\
12\end{array}$ & $\begin{array}{l}47.4 \% \\
10.5 \%\end{array}$ & $\begin{array}{l}25 \\
13\end{array}$ & $\begin{array}{l}30.8 \% \\
16.0 \%\end{array}$ & 104 \\
\hline Total & & 114 & & 81 & & 195 \\
\hline
\end{tabular}

$p$ value was calculated by two independent sample test (Mann-Whitney Test)

${ }^{\text {a }}$ Significant variant growth pattern distribution being found (vs. children patients, $p=0.023$ )

${ }^{\mathrm{b}}$ Infra-diaphragmatic CPs predominantly distributed in children cases $(p<0.0001)$

${ }^{\mathrm{c}}$ Extra-ventricular CPs were observed predominantly in adult patients $(p<0.0001)$

${ }^{\mathrm{d}}$ Infundibulo-tuberal CPs with pure pattern "Sa" distributed preferable in adult cases $(p=0.021)$

axial route is most appropriate, used either alone or in conjunction with the extra-axial route. This is because tumor grows within the third VF without any separation of the arachnoid.

Of note, our classification is based on anatomical growth models and therefore should not be used as a replacement of other pre-existing classifications (e.g., Yasargil, Hoffman and Kassam et al.). It should instead be viewed as a supplement to these in view of the complex clinical and morphological features of CP. It is inadequate to describe tumor growth only through an analysis of the membranous structures. In this paper, we list the 195 cases CPs divided into infra-diaphragmatic, extra-ventricular, extra- and intraventricular, trans-infundibular and tubero-infundibular tumors. This was based on previous classifications. However, in using our method to explain the growth pattern of CPs, it allows for greater understanding of tumor growth and intraoperative stratification. Even CPs with highly variable appearances can still be understood using our four basic growth models.

\section{Distribution in children and adult patients}

There has been no previously published data noting the variable growth patterns of CPs in adults versus children. However, through review of the literature, some patterns can be identified. Gabriel L et al. [19] reported their experience in treating 47 children with CP. They divided these into four types-14 purely intrasellar cases (29.8\%), 25 tuberoinfundibular cases $(53.2 \%), 1$ strictly intra-ventricular case (2\%), and 7 classed as global or giant cases (14.9\%). Yi-Yen Lee et al. [18] used Choux-Raybaud's [3] classification to differentiate 66 pediatric CPs into 27 purely intrasellar tumors (40.9\%) and 39 third ventricular (infundibulum and tuber cinereum) tumors (59.1\%) through MRI, CT or operative findings. Interestingly, no extra-ventricular tumors were reported in these cohorts. All these data based on morphological features of these tumors in the pediatric population indicated a paucity of the extra-ventricular type in young patients. This finding has been replicated in this study. Following statistical analysis, only 3 among the 81 children with CPs $(3.7 \%)$ proved to have the extra-ventricular subtype, indicating a much lower incidence compared to the $28.1 \%(32 / 114)$ observed in adult patients.

On the other hand, infra-diaphragmatic tumors were predominantly observed in children $(42.0 \%$ vs. $7.0 \%)$. Although no literature specifically addresses CPs in adults, through a review of the literature [1, 15-17] on early use of the transsphenoidal approach to treat CPs, it was noted that most of the patients were young and mainly had the sub-diaphragmatic subtype of tumor. Recently, with the endoscope being applied to the supra-diaphragmatic transsphenoidal approach, there has been an emphasis on adult patients with CPs in several papers [4-6, 11, 12]. In 2010, Jane et al. [11] reported their experience of endoscopy in treating 12 cases of adult CPs. These were exclusively the suprasellar subtype, and did not include any sub-diaphragmatic subtypes.

Additionally, although no significant different was found in the incidence of the tubero-infundibular tumors between children and adults, our study showed a predominance of the purely "Sa" growth pattern tumors, in adults $(47.4 \%$ vs. $30.8 \%$ ). Purely pattern "Sa" tumors have a site of origin from the infundibulum and tuber cinereum with the tumor nest being within the third ventricular floor, growing upwards to the ventricular cavity without 
extension to the extra-ventricular region or within the pituitary stalk. These are common features seen in intraventricular CPs. In keeping with pre-existing published work, we observed that intra-ventricular CPs occurred at an older age $[2,9,22,23]$.

In summary, clinical analysis of this cohort (Table 1) displayed a statistically different distribution between adults and children $(p=0.023)$. Our results not only indicated a variant growth pattern of CPs according to age, but also hinted that membranous structures (such as the diaphragm and ASPS) may play roles in directing tumor growth.

\section{Conclusions}

An arachnoidal sleeve was found enveloping the PS, directly inducing segmental division of the stalk and leading to our proposed theory of four basic growth pattern of CP (ID, EA, IA, and SA). Each type of tumor growth shows totally different morphological features and tumor locations. Consequently, there are implications on the choice of surgical technique and efforts to protect the PS and third VF. Although we acknowledge that the growth model of CPs is highly complex and variable, our study attempts to provide some insight into an explanation, with the aid of intraoperative observations. The tumor-membrane relationship is clearly identified and this in turn gives a direct impression of the surgical stratification of these tumors. This in turn has clear implications on the surgical management. As a supplement to existing classification schemes for CPs, our topographic division of CPs is intended to be of benefit in better understanding the growth pattern of these tumors.

Acknowledgment The editorial office would like to thank Dr. Apok for the editing and rewriting of the English language

Conflicts of interest This study is financially supported by the Chinese national natural science funding (No. 81072067) and the Guangdong province natural science funding (No. 9451051501003989), China

Open Access This article is distributed under the terms of the Creative Commons Attribution Noncommercial License which permits any noncommercial use, distribution, and reproduction in any medium, provided the original author(s) and source are credited.

\section{References}

1. Abe T, Ludecke DK (1999) Transnasal surgery for infradiaphragmatic craniopharyngiomas in pediatric patients. Neurosurgery 44:957-964, discussion 964-956

2. Behari S, Banerji D, Mishra A, Sharma S, Chhabra DK, Jain VK (2003) Intrinsic third ventricular craniopharyngiomas: report on six cases and a review of the literature. Surg Neurol 60:245-252, discussion 252-243
3. Brunel H, Raybaud C, Peretti-Viton P, Lena G, Girard N, PazParedes A, Levrier O, Farnarier P, Manera L, Choux M (2002) Craniopharyngioma in children: MRI study of 43 cases. NeuroChirurgie 48:309-318

4. Campbell PG, McGettigan B, Luginbuhl A, Yadla S, Rosen M, Evans JJ (2010) Endocrinological and ophthalmological consequences of an initial endonasal endoscopic approach for resection of craniopharyngiomas. Neurosurg Focus 28:E8

5. Dusick JR, Esposito F, Kelly DF, Cohan P, DeSalles A, Becker DP, Martin NA (2005) The extended direct endonasal transsphenoidal approach for nonadenomatous suprasellar tumors. J Neurosurg 102:832-841

6. Frank G, Pasquini E, Doglietto F, Mazzatenta D, Sciarretta V, Farneti G, Calbucci F (2006) The endoscopic extended transsphenoidal approach for craniopharyngiomas. Neurosurgery 59:ONS75-ONS83

7. Hoffman HJ (1994) Surgical management of craniopharyngioma. Pediatr Neurosurg 21(Suppl 1):44-49

8. Hoffman HJ, De Silva M, Humphreys RP, Drake JM, Smith ML, Blaser SI (1992) Aggressive surgical management of craniopharyngiomas in children. J Neurosurg 76:47-52

9. Ikezaki K, Fujii K, Kishikawa T (1990) Magnetic resonance imaging of an intraventricular craniopharyngioma. Neuroradiology 32:247-249

10. Inoue $\mathrm{K}$, Seker A, Osawa S, Alencastro LF, Matsushima $\mathrm{T}$, Rhoton AL (2009) Microsurgical and endoscopic anatomy of the supratentorial arachnoidal membranes and cisterns. Neurosurgery 65:644-664, discussion 665

11. Jane JA Jr, Kiehna E, Payne SC, Early SV, Laws ER Jr (2010) Early outcomes of endoscopic transsphenoidal surgery for adult craniopharyngiomas. Neurosurg Focus 28:E9

12. Jane JA Jr, Prevedello DM, Alden TD, Laws ER Jr (2010) The transsphenoidal resection of pediatric craniopharyngiomas: a case series. J Neurosurg Pediatr 5:49-60

13. Kassam AB, Gardner PA, Snyderman CH, Carrau RL, Mintz AH, Prevedello DM (2008) Expanded endonasal approach, a fully endoscopic transnasal approach for the resection of midline suprasellar craniopharyngiomas: a new classification based on the infundibulum. J Neurosurg 108:715-728

14. Kitano M, Taneda M (2009) Extended transsphenoidal surgery for suprasellar craniopharyngiomas: infrachiasmatic radical resection combined with or without a suprachiasmatic translamina terminalis approach. Surg Neurol 71:290-298, discussion 298

15. Konig A, Ludecke DK, Herrmann HD (1986) Transnasal surgery in the treatment of craniopharyngiomas. Acta Neurochir (Wien) 83:1-7

16. Laws ER Jr (1980) Transsphenoidal microsurgery in the management of craniopharyngioma. J Neurosurg 52:661-666

17. Laws ER Jr (1994) Transsphenoidal removal of craniopharyngioma. Pediatr Neurosurg 21(Suppl 1):57-63

18. Lee YY, Wong TT, Fang YT, Chang KP, Chen YW, Niu DM (2008) Comparison of hypothalamopituitary axis dysfunction of intrasellar and third ventricular craniopharyngiomas in children. Brain Dev 30:189-194

19. Lena G, Paz Paredes A, Scavarda D, Giusiano B (2005) Craniopharyngioma in children: Marseille experience. Childs Nerv Syst 21:778-784

20. Lu J, Zhu XL (2005) Characteristics of distribution and configuration of intracranial arachnoid membranes. Surgical and radiologic anatomy: SRA 27:472-481

21. MG Y (1984) Microneurosurgery. Thieme Verlag 1:5-53

22. Pascual JM, Carrasco R, Prieto R, Gonzalez-Llanos F, Alvarez F, Roda JM (2008) Craniopharyngioma classification. J Neurosurg 109:1180-1182, author reply 1182-1183

23. Pascual JM, Gonzalez-Llanos F, Barrios L, Roda JM (2004) Intraventricular craniopharyngiomas: topographical classification 
and surgical approach selection based on an extensive overview. Acta Neurochir (Wien) 146:785-802

24. Samii M, Bini W (1991) Surgical treatment of craniopharyngiomas. Zentralbl Neurochir 52:17-23

25. Samii M, Tatagiba M (1997) Surgical management of craniopharyngiomas: a review. Neurol Med Chir 37:141-149

26. Song-tao Q, Xi-an Z, Hao L, Jun F, Jun P, Yun-tao L (2010) The arachnoid sleeve enveloping the pituitary stalk: anatomical and histologic study. Neurosurgery 66:585-589

27. Songtao Q, Yuntao L, Jun P, Chuanping H, Xiaofeng S (2009) Membranous layers of the pituitary gland: histological anatomic study and related clinical issues. Neurosurgery 64:1-9, discussion $9-10$

28. Steno J, Malacek M, Bizik I (2004) Tumor-third ventricular relationships in supradiaphragmatic craniopharyngiomas: corre- lation of morphological, magnetic resonance imaging, and operative findings. Neurosurgery 54:1051-1058, discussion $1058-1060$

29. Wang KC, Hong SH, Kim SK, Cho BK (2005) Origin of craniopharyngiomas: implication on the growth pattern. Childs Nerv Syst 21:628-634

30. Wang KC, Kim SK, Choe G, Chi JG, Cho BK (2002) Growth patterns of craniopharyngioma in children: role of the diaphragm sellae and its surgical implication. Surg Neurol 57:25-33

31. Yasargil MG, Curcic M, Kis M, Siegenthaler G, Teddy PJ, Roth P (1990) Total removal of craniopharyngiomas. Approaches and long-term results in 144 patients. J Neurosurg 73:3-11

32. Yasargil MG, Kasdaglis K, Jain KK, Weber HP (1976) Anatomical observations of the subarachnoid cisterns of the brain during surgery. J Neurosurg 44:298-302 